Hydroécol. Appl. (1993) Tome 5 Vol. 2, pp. 43-60

\title{
Gestion des plantes aquatiques dans les lacs et les étangs landais
}

\section{Management of aquatic weeds in lakes and ponds of the Landes (France)}

\author{
Alain Dutartre ${ }^{\star}$, Joachim Oyarzabal ${ }^{\star \star}$ \\ * CEMAGREF, Division Qualité des Eaux, BP 3, 33611 Gazinet cedex \\ * Conseil Général des Landes, Direction Aménagement "Espaces Naturels", 20-22 rue Victor \\ Hugo, 40025 Mont de Marsan cedex.
}

Résumé. - Le climat océanique très doux de la région Aquitaine favorise l'installation d'espèces végétales exotiques, en provenance de régions d'autres continents aux climats tempérés ou subtropicaux. Ce phénomène relativement récent est observable dans la région depuis 25 à 30 ans. Trois espèces ont considérablement envahi la frange de plans d'eau du littoral où elles engendrent des difficultés variables, telles que des nuisances vis à vis des usages des milieux ou une banalisation écologique des secteurs colonisés.

Devant l'augmentation des difficultés créées par ces espèces, une étude destinée à faire un bilan de la situation et à établir un plan de gestion a été réalisée en 1988-1989. Une approche cartographique a permis de mieux analyser la répartition de ces espèces et leurs modes de colonisation des milieux. Le plan de gestion comportait des interventions "urgentes", des essais de techniques comme l'emploi de moissonneurs aquatiques et d'herbicides, et des efforts de formation destinés à favoriser des interventions sur les milieux en début "d'invasion".

Depuis 1988, diverses opérations destinées à contrôler les proliférations végétales jugées nuisibles ont été entreprises. Certaines d'entre elles sont des interventions directes sur les plantes, comme une moisson aquatique ou des essais d'herbicides, d'autres, comme par exemple des dragages ou une mise en assec, sont des travaux modifiant l'habitat des plantes. L'examen de ces opérations permet de dresser un premier bilan des moyens techniques disponibles, des difficultés d'organisation et de mise en œuvre d'une gestion des plantes aquatiques dans des plans d'eau multi-usages.

Mots-clés. - Flore aquatique - Contrôle de la végétation - Gestion des milieux aquatiques - Plans d'eau. 


\begin{abstract}
The oceanic mild weather of Aquitaine (France) promotes growth of exotic plants species native from temperate and subtropical areas of the world. This quite recent phenomenom has been observed in Aquitaine for 25 to 30 years. Three species have considerably invaded the littoral lakes where they are responsible of variable difficulties, such as water uses nuisances or ecological banalisation of colonized sectors.

In 1988-1989, a study was conducted to evaluate this difficulties and to establish a weed management plan. Maps were made to better understand weed distribution and colonization modes. The management plan proposed urgent interventions and efforts of people formation allowing interventions on recently invaded lakes.

Different interventions for controlling noxious weed infestations have been undertaken for 1988. Some of them have direct effects on plants (plant harvesting, herbicide treatments), others change plant habitat (dredging, emptying and drying).

The evaluation of these operations allows to strike a first balance of available control techniques and of difficulties of organization of water plant management in multi-uses lakes and ponds.
\end{abstract}

Key words. - Aquatic plant - Vegetation control - Water bodies management.

\section{INTRODUCTION}

La colonisation progressive des biotopes par les espèces végétales et animales est un processus continu et naturel que l'homme a utilisé et continue d'utiliser pour ses propres besoins. Cette colonisation peut concerner des biotopes nouveaux, tels que les îles volcaniques ou les zones abandonnées par les glaciers ou des biotopes dégradés ou totalement modifiés, soit par des catastrophes naturelles, soit par des actions humaines.

Depuis la généralisation des échanges commerciaux intercontinentaux, de très nombreuses especes ont été transportées sciemment ou accidentellement; les premières sont cultivées ou élevées et leur maintien est lié aux activités humaines, les secondes ont pu, dans de nombreux cas, s'installer dans des biotopes favorables et, en l'absence de toute ré- gulation (prédateurs, pathogènes,...), ont pu ensuite proliférer et causer des dommages et des nuisances. De tels "envahisseurs" sont bien connus parmi les animaux et les végétaux. En ce qui concerne les plantes, les exemples les plus spectaculaires sont ceux de la jacinthe d'eau (Eichhornia crassipes) et de l'élodée du Canada (Elodea canadensis) (Sculthorpe, 1967).

Ces invasions sont particulièrement évidentes dans les plans d'eau douce du littoral aquitain (figure $n^{\circ} 1$ ) dont le climat océanique très doux en fait une région très accueillante pour des plantes aquatiques exotiques. A cela vient s'ajouter la grande diversité des types de milieux aquatiques (lacs, étangs, marais, etc...),

Parmi ces espèces introduites, deux ont une extension actuelle importante et, pour certains plans d'eau, très préoccupante: il s'agit d'une espèce immergée, le lagarosiphon ( $L a-$ garosiphon major) et d'une espece 


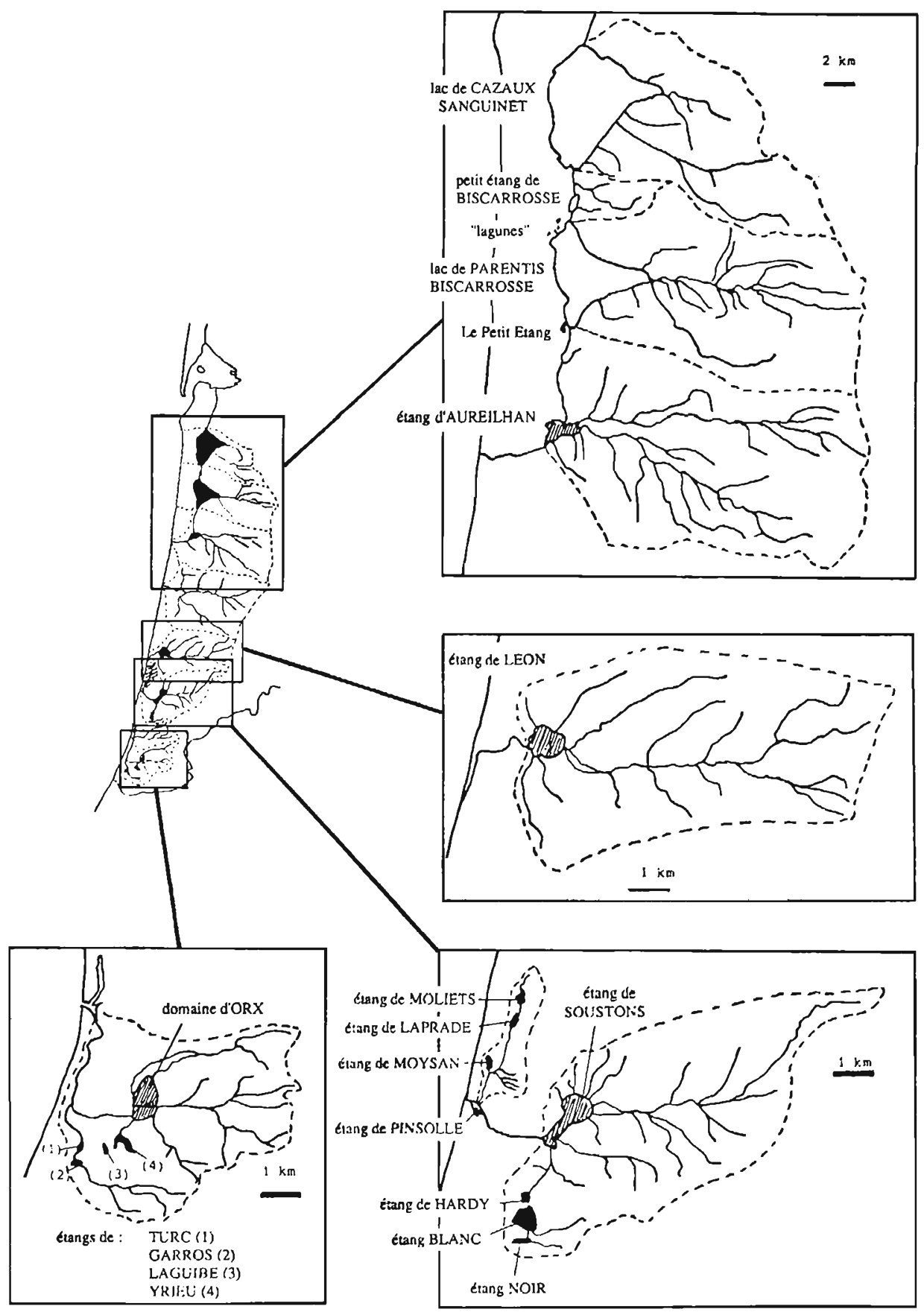

Fig. 1. - Situation des plans d'eau du littoral landais. 
amphibie, c'est à dire se développant aussi bien dans l'eau qu'en dehors, la jussie (Ludwigia peploïdes ou $L$. urugayensis). Une troisième espèce, amphibie elle aussi, le myriophylle du Brésil (Myriophyllum brasiliense) a une extension notable mais semble poser actuellement moins de problèmes.

II s'agit d'un phénomène que l'on peut considérer comme récent : la jussie a été introduite au début du siècle mais son extension n'est devenue visible que depuis deux décennies, les deux autres espèces ont été observées seulement depuis 25 à 30 ans. L'écologie, les origines, et le mode de propagation de ces espèces sont relativement bien connus (Dutartre, 1978). Elles n'ont occupé tout d'abord que des secteurs restreints de certains plans d'eau, puis elles ont pris de l'extension, lentement dans quelques cas, plus rapidement dans d'autres. Elles sont actuellement présentes dans la majeure partie des plans d'eau (tableau $n^{\circ} 1$ ); leur développement est en progression continue, mais avec des vitesses variables selon les espèces et les types de milieux.

D'autres espèces, telles que la petite fougère Azolla filiculoïdes et l'hibiscus (Hibiscus roseus) sont présentes mais ne constituent au pire que des gênes locales et de durée limitée.

Les espèces introduites ne peuvent être considérées systématiquement comme nuisibles; en effet elles occupent quelquefois des biotopes non colonisés par les plantes «indi- gènes ", elles peuvent alors jouer un rôle de diversification du milieu et des habitats disponibles pour la faune. En revanche, dans d'autres cas elles concurrencent les plantes indigènes. Leur rôle devient alors négatif: on assiste alors à une certaine banalisation du milieu conduisant à une baisse de sa richesse écologique.

Les espèces "indigènes" ont en général des extensions plus réduites et un développement plus lent. Les secteurs qu'elles occupent sont le plus souvent de dimensions restreintes, en "couronne" ou en "ceinture" autour du plan d'eau, selon les différents gradients de profondeur (Dulong, Dutartre, 1989). Dans d'autres cas, les modifications de milieu, comme par exemple le comblement des plans d'eau par les sables et les vases, fournissent à ces espèces de nouveaux territoires et l'évolution qui en résulte est facilement remarquable par les utilisateurs habituels du plan d'eau.

Les facteurs de climat et d'habitat expliquent également la présence d'espèces rares ou endémiques, protégées ou non, qui constituent des éléments non négligeables du patrimoine floristique régional. Les activités humaines développées sur les plans d'eau ou leurs bassins versants, dont le tourisme estival, font peser des menaces importantes sur ces espèces, soit directement par la raréfaction des habitats favorables, soit indirectement par l'eutrophisation des eaux.

Les nuisances occasionnées par les proliférations végétales dépendent 
Tableau 1. - Récapitulatif des premières observations des plantes introduites (observations personnelles, communications personnelles diverses)

\begin{tabular}{|c|c|c|c|}
\hline Plan d'eau & Lagarosiphon & Jussie & Myriophylle \\
\hline Cazaux-Sanguinet & environ 1965 & 1979 & \\
\hline Biscarrosse & 1978 & 1978 & \\
\hline Parentis-Biscarrosse & 1976 & avant 1976 & \\
\hline Aureilhan & 1978 & 1978 & \\
\hline Léon & 1991 & 1988 & \\
\hline Moliets & & 1991 & 1953 \\
\hline Laprade & & $?$ & 1953 \\
\hline Moīsan & & & 1953 \\
\hline Soustons & 1986 & $?$ & $?$ \\
\hline Hardy & 1986 & $?$ & $?$ \\
\hline Blanc & 1983 & 1986 & $?$ \\
\hline Noir & & 1983 & 1983 \\
\hline Turc & & 1982 & \\
\hline Garros & & 1988 & \\
\hline Orx & & & \\
\hline Yrieu & & & \\
\hline
\end{tabular}

Tableau 1. - Eventualité de la présence de l'espèce, car aval de plans d'eau déjà touchés

de la localisation de l'espèce dans le milieu (si elle occupe les rives ou la profondeur), de la taille relative du milieu et du secteur colonisé (un herbier de dimensions réduites peut être gênant dans un petit étang mais sera insignifiant dans un des lacs du Nord du département) et enfin de la nature et de l'intensité des usages développés sur ce milieu (Dutartre et al, 1986). En effet, il faut rappeler qu'une nuisance est une gêne occasionnée à un usage humain du milieu (notion essentiellement anthropique): dans un plan d'eau que I'homme n'utilise pas, il n'y a pas de nuisances...

Ces nuisances sont principalement des difficultés de déplacement des baigneurs, des embarcations à voile ou à moteur: les herbes viennent se prendre dans les dérives ou les hélices... La pêche est également gênée dans la mesure où il devient difficile de pêcher dans des herbiers denses: 
même si de nombreux poissons s'y trouvent, ils sont quasiment impossibles à prendre.

Le développement de la végétation aquatique, constituée soit d'algues, soit de végétaux macrophytes, modifie la physionomie des milieux et leurs attraits pour les utilisateurs: l'esthétique des milieux et leur attrait paysager peuvent souffrir de ces proliférations végétales lorsqu'elles sont le fait d'une seule espèce qui banalise le paysage; dans la région, ce n'est pas un aspect négligeable.

\section{GESTION DES PLANTES AQUATIQUES}

Les plans d'eau sont, pour la plupart, communaux; ils peuvent se trouver sur le territoire d'une seule ou de plusieurs communes, ce qui ne facilite pas toujours la mise en place d'une gestion à l'échelle du milieu. Depuis la fin des années 70 , devant la progression des proliférations dans certains plans d'eau, nous avons été amenés à réaliser des campagnes d'observations et à rédiger divers rapports d'étude ou notes, destinées à attirer l'attention des gestionnaires sur ces difficultés de gestion. Mais il a fallu la création du Syndicat Mixte Géolandes ${ }^{(1)}$ pour que cet aspect de la

(1) Syndicat réunissant le Conseil Général des Landes et 19 communes riveraines des plans d'eau. gestion des plans d'eau soit effectivement pris en compte. Lors de l'enquête menée pour ce syndicat auprès des communes riveraines des lacs et des étangs (Dutartre et al, 1989), la maîtrise du développement (ponctuel ou général) de végétaux macrophytes a été citée comme nécessaire sur la majorité des plans d'eau, illustration évidente des besoins en la matière. Un plan de gestion a été proposé dans le même document pour tenter d'aborder de manière coordonnée ces difficultés. II a déjà fait l'objet d'informations sur son contenu (Dulong, Dutartre, 1989 et Dutartre, 1989). II prévoyait plusieurs actions destinées à lutter contre les proliférations les plus problématiques, à tester l'emploi d'herbicides dans certains cas et à mettre en place des opérations d'information et de formation.

\subsection{Choix techniques}

Les raisons des choix préliminaires parmi les techniques mécaniques, chimiques, biologiques et de modifications des habitats ont été expliquées dans divers documents (Dulong, Dutartre, 1989 et Dutartre, 1989), mais peuvent se résumer en un critère simple: "contrôler le moyen de contrôle". En effet, compte tenu de l'extrême imbrication des usages dans ces plans d'eau, il nous paraissait indispensable de limiter l'extension spatiale dans les milieux des techniques employées.

C'est pourquoi les solutions biologiques ont été écartées dans un pre- 
mier temps : certaines d'entre elles ne nous semblaient pas offrir des garanties de contrôle ultérieur, comme l'emploi de la carpe chinoise, d'autres restaient encore au stade théorique ou en phase de préexpérimentation, comme l'utilisation de pathogènes ou de consommateurs spécifiques; à cette époque, nous n'avions d'ailleurs pas trouvé de références de travaux de recherche dans ce domaine, consacrés à Lagarosiphon ou à Ludwigia.

Les techniques utilisées pour contrôler ces proliférations sont de plusieurs types:

- contrôle mécanique par moisson ou arrachage,

- traitements à l'aide d'herbicides,

- dragage; cette technique ne vise pas directement la prolifération des plantes mais permet un contrôle souvent efficace car elle permet, en enlevant les couches superficielles des sédiments, de retirer aussi du milieu racines et tiges des plantes.

- mise en assec et décapage des parties superficielles des sédiments.

Le tableau $n^{0} 2$ récapitule les travaux déjà effectués (Dutartre, sous presse)

\subsection{Contrôle mécanique}

\subsubsection{Travaux sur l'Etang Blanc}

Les travaux de faucardage et de moisson de $L$. major ont débuté en 1988 et sont réalisés annuellement pour dégager des zones utilisables par les loisirs nautiques (voile, planche à voile,...) (tableau $n^{\circ} 3$ ).

En 1988, les premiers travaux avaient été réalisés à l'aide d'un bateau faucardeur classique, auquel a été adjoint un râteau de récolte relevable. Des quantités difficiles à estimer de plantes coupées sont restées dans le milieu. Dans le plan de gestion proposé à Géolandes en 1989, nous avions insisté sur la nécessité de réaliser une moisson véritable, c'est à dire coupe et récolte simultanées. Aussi les années suivantes, les travaux ont-ils été effectués à l'aide d'un bateau moissonneur, permettant de pratiquer cette moisson.

En 1989, des essais comparatifs ont été faits pour choisir l'une des deux sociétés possédant ce matériel en France. En 1990 et 1991, des opérations en vraie grandeur ont été mises en place. Ce sont des opérations coûteuses et répétitives. Une recherche d'optimisation de ces travaux a conduit à les réaliser de la manière suivante :

- le bateau moissonneur travaille dans des zones de dimensions réduites, où ses déplacements ne sont pas trop longs,

- quatre conteneurs transportables par camions sont chargés sur deux barges, l'une toujours proche de la zone moissonnée,

- le moissonneur décharge périodiquement les plantes coupées dans les deux conteneurs présents sur la zone de travaux,

- lorsque les conteneurs sont pleins, ils sont transportés vers le 
Tableau 2. - Actions de contrôle des plantes aquatiques dans les lacs et étangs du littoral landais

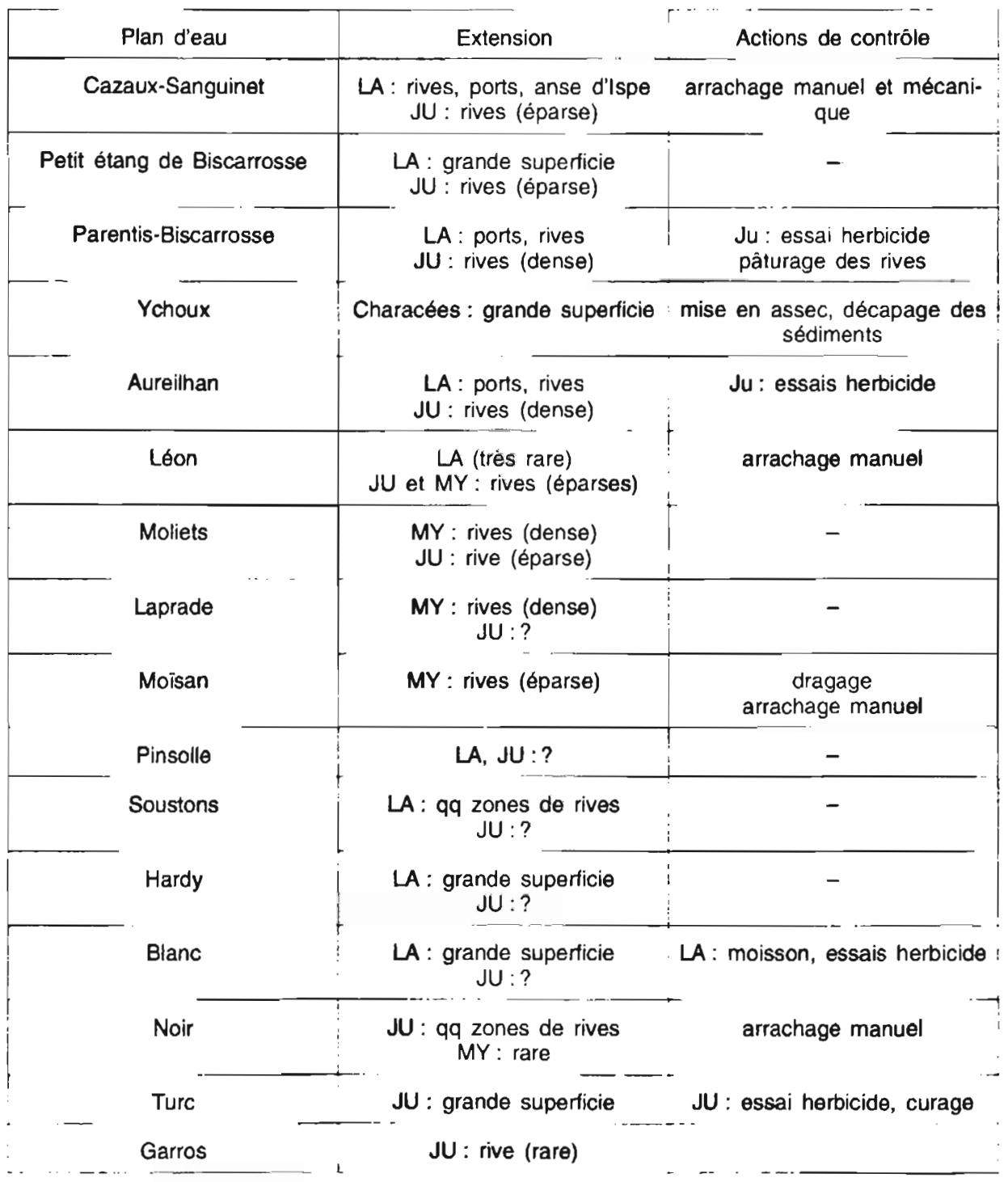

Espèces introduites: LA : lagarosiphon, JU : jussie, MY : myriophylle du Brésil 
Tableau 3. - Traitements mécaniques du l'étang blanc.

\begin{tabular}{|c|c|c|c|}
\hline Année & Superficie (ha) & Volume Extrait $\left(\mathrm{m}^{3}\right)$ & Coût (TTC) \\
\hline 1988 & $?$ & $?$ & 47000 \\
\hline 1989 & $3,7^{*}$ & 880 & 125000 \\
\hline 1990 & 50 & 13000 & 900000 \\
\hline 1991 & 28 & 7750 & 500000 \\
\hline 1992 & 23 & 6365 & 338000 \\
\hline
\end{tabular}

- Essais comparatifs

quai, spécialement construit à cet effet, et chargés sur des camions; la barge portant les conteneurs vides est ramenée vers la zone de travaux.

Les plantes récoltées ont été transportées sur des terrains agricoles proches et utilisées comme engrais vert par un seul agriculteur d'abord, puis plusieurs, ce qui a évité un transport plus long vers une décharge autorisée.

\subsubsection{Travaux sur l'Etang du Turc}

Devant l'extrême colonisation de cet étang par Ludwigia, nous avions proposé comme moyen de contrôle de réaliser un arrachage complet des herbiers. Cette technique a été mise en place à automne 1992 (les travaux sont actuellement en cours). Une griffe montée sur un bras de pelle hydraulique est installée sur une barge équipée de deux flotteurs latéraux lui permettant une meilleure stabilité. Cette griffe assure l'arrachage de l'ensemble des plantes, systèmes racinaires compris. Compte tenu des superficies importantes couvertes par l'espèce dans l'étang, l'engin travaille en bandes successives d'une dizaine de mètres de largeur, de la rive vers le large; le chargement des masses végétales s'effectue lorsque qu'elles sont entassées en cordon, dans des conteneurs transportables par remorque derrière un tracteur agricole, pour un dépôt ultérieur proche.

Nous avons prévu de réaliser au printemps 1993, une campagne d'observations sur la présence de la plante dans l'étang afin de proposer des interventions plus limitées d'entretien régulier, destinées à contrôler la recolonisation du plan d'eau.

\subsubsection{Autres travaux}

Depuis 1978, divers travaux d'arrachage ont été réalisés dans quelques plans d'eau, dont en particulier le lac de Cazaux-Sanguinet. Ils concernaient une anse de 5 ha située sur la rive sud ouest du lac où $L$. major se développe dans des eaux limpides et calmes depuis 4 mètres de profondeur, jusqu'en surface (Dutartre, 1979), et une Halte Nautique de création récente au nord ouest, très rapidement envahie par la même espèce. 
Dans le premier site, l'arrachage a été fait en 1978 à l'aide de câbles tirés depuis la berge, plus récemment à l'aide d'un moissonneur. Dans le second site, fortement occupé par les installations portuaires et les bateaux à quai, l'arrachage a été fait manuellement par des plongeurs autonomes.

\subsection{Herbicides}

L'objectif de ces traitements expérimentaux était d'analyser les possibilités de traitements complémentaires sur des zones déjà traitées mécaniquement, ou de traitements seuls sur des zones limitées où une totale absence de plantes est souhaitable. Un suivi des impacts sur l'environnement a été réalisé dans certains de ces essais; il comportait des examens de la qualité des eaux, des réactions des algues in situ et des tests de toxicité en laboratoire. Dans tous les cas, ces suivis ont montré de très faibles variations de ces paramètres, dans la mesure où les essais étaient de superficie limitée dans des zones envahies de grandes dimensions. Nous ne présenterons donc ici que les résultats en terme d'efficacité de contrôle.

\subsubsection{Essais sur Lagarosiphon}

En 1989, trois produits ont été testés sur des zones de superficie limitée de l'Etang Blanc. II s'agit de Aquaprop (produit homologué dont la matière active est du dichlobénil), de Aquathol et de Sonar P5 (matieres actives : endothall et fluridone, ces deux derniers produits n'étaient pas homologués à l'époque).
Les résultats ont été nuls pour Aquaprop, très peu probants pour le Sonar et bons pour Aquathol.

Lors des essais avec le Sonar, des effets phytotoxiques ont été observés dans la zone traitée, mais au bout de quelques semaines ils ont disparus et les plantes sont restées en place. Comme cet essai faisait partie d'une batterie de tests mise en place par la société Dowelanco, préalablement à I'homologation de ce produit en France, et qu'il était le seul à présenter cette particularité, nous avons réalisé en 1990 des essais de laboratoires destinés à préciser les raisons de cette absence d'efficacité. Les résultats obtenus montrent qu'une partie non négligeable du produit reste non actif dans les vases organiques (Dubernet et al, 1992); il est également probable que l'espèce présente une résistance particulière à ce produit.

Les essais avec Aquathol ont montré l'efficacité du produit: dans la zone traitée les plantes avaient disparu, seul un dépôt très fluide à la surface des sédiments vaseux subsistait quelques semaines après le traitement. Nous avons suivi la recolonisation de cette zone par le lagarosiphon en réalisant des prélèvements de plantes en 1989, 1990 et 1991 (tableau $n^{\circ} 4$ ), sachant que la biomasse moyenne (mesurée après prélèvements en plongée de quadrats de $0,25 \mathrm{~m}^{2}$, données non publiées) des zones proches non traitées dépasse $1000 \mathrm{~g}$ de matières sèches $/ \mathrm{m}^{2}$. 
Tableau 4. - Evolution de la biomasse de Lagarosiphon dans la zone traitée avec endothall

\begin{tabular}{|c|c|}
\hline Année & (en g de mat. sèches $/ \mathrm{m}^{2}$ ) \\
\hline $\begin{array}{c}1989 \\
1990\end{array}$ & $\frac{13}{40}$ \\
\hline 1991 & $-\frac{103}{103}$ \\
\hline
\end{tabular}

\subsubsection{Essais sur Ludwigia}

Un premier essai a été réalisé en 1973 sur un herbier dense de Ludwigia à proximité du port de Parentis (Anonyme, 1975): la matière active employée était du diquat, herbicide de contact. Si la plante a bien été détruite par le traitement, la repousse à partir des bases immergées des tiges non directement touchées par l'aérosol s'est fait dans les mois qui ont suivis, annulant de ce fait les résultats du départ.

Des essais ont été fait en 1988 et 1989 sur quelques secteurs de rives colonisés de l'Etang du Turc et de l'Etang d'Aureilhan, avec assez peu de résultats. Les essais avec de l'Aquaprop n'ont montré qu'une efficacité de courte durée, puisque, après des effets phytocides très nets, la repousse de l'année suivante dans les zones traitées ne montrait, comme indices des effets du traitement, qu'une diminution de taille moyenne des tiges hors de l'eau et une raréfaction des fleurs.
Un essai avec Aquathol n'a montré aucun effet visible.

Des essais réalisés en 1988 par une société spécialisée, à l'aide de trois produits, ont donnés des résultats variables la première année, très faibles à la saison suivante (tableau $\left.n^{\circ} 5\right)$ : si, dans certains cas, les résultats ont perduré pendant l'année suivant les applications de produit, plus rien n'était visible en 1990. Devant la colonisation très importante de ce milieu, seule des techniques mécaniques radicales restaient possibles, d'où nos propositions (voir paragraphe 2.2.2.)

\subsection{Autres interventions}

\subsubsection{Dragage de l'Etang de Moïsan}

L'étang de Moïsan est un plan d'eau d'environ 6 ha et dont la hauteur d'eau avant travaux était quasiment nulle en été. A la suite d'une étude de ce milieu (Collectif, 1985), comme des solutions de restauration étaient souhaitées, nous avions proposé un dragage sur l'ensemble de sa superficie. Ces travaux ont été réalisés en 1990-1991 et ont comporté un enlèvement de sables et de vases organiques destiné à redonner à ce plan d'eau une profondeur proche de 1,5 m. II était fortement colonisé par $M$. brasiliense et d'autres espèces "indigènes" fortement limitées par cette prolifération et par celles de lentilles d'eau (Lemna minor et L. gibba) et de la fougère Azolla filiculoïdes: le 
Tableau 5. - Essais réalisés sur la Jussie dans l'étang du Turc

Le traitement a été réalisé en août 1988.

La gamme de note utilisée s'étend de 0 (aucun effet visible) à 9 (disparition totale des plantes).

\begin{tabular}{|c|c|c|c|c|c|}
\hline \multirow{3}{*}{$\begin{array}{c}\text { Essais } \\
1\end{array}$} & \multirow{3}{*}{$\begin{array}{l}\text { Produit } \\
\text { Roundup } 360\end{array}$} & \multirow[b]{2}{*}{ Dosage } & \multicolumn{2}{|c|}{ Observations } & \multirow[b]{2}{*}{$08 / 1990$} \\
\hline & & & $10 / 1988$ & $08 / 1989$ & \\
\hline & & $9 \mathrm{l} / \mathrm{ha}$ & 7 & 2 & 0 \\
\hline 2 & Roundup 360 & $12 \mathrm{l} / \mathrm{ha}$ & 6 & 2 & 0 \\
\hline 3 & 'Roundup "dilué" & $36 \mathrm{~g} / \mathrm{ha}$ & 7 & 2 & 0 \\
\hline 4 & Roundup "diluén & $48 \mathrm{~g} / \mathrm{ha}$ & 8 & 3 & 0 \\
\hline 5 & Rhizol El & $40 \mathrm{l} / \mathrm{ha}$ & 6 & 4 & 0 \\
\hline 6 & Roundup 360 & 12 l/ha & 5 & 4 & 0 \\
\hline 7 & Rhizol El & $40 \mathrm{l} / \mathrm{ha}$ & 6 & 3 & 0 \\
\hline
\end{tabular}

N.B. : Les matières actives des produits commerciaux appliqués sont du glyphosate pour le Roundup et aminotriazole + thiocyanate d'ammonium pour le Rhizol.

dragage a également enlevé ces macrophytes.

\subsubsection{Traitement de la retenue d'Ychoux}

II s'agit d'un plan d'eau artificiel créé par barrage d'un des affluents du lac de Parentis-Biscarrosse. Depuis plusieurs années, une prolifération de Characées gênait la pratique de la pêche dans cette retenue de 3 hectares. En 1987, un essai à l'aide d'un bateau moissonneur n'avait pas donné les résultats escomptés: les macroalgues n'étaient pas coupées puis montées sur le tapis roulant, mais seulement entassées devant l'engin. Un assec, suivi d'un décapage des couches superficielles des sédiments à la dragline a donc été fait en réalisé en 1992. Un suivi devra être fait au printemps 1993 pour analyser l'évolution des peuplements de macrophytes du milieu: à l'heure actuelle des herbiers de Potamogeton natans sont en train de recoloniser rapidement une grande partie du plan d'eau.

\subsection{Formation et information}

\subsubsection{Stage 1991}

Au printemps 1991, avec un financement du Syndicat Mixte Géolandes, nous avons réalisé un stage de formation suivi de visites de terrain "spécialisées" sur divers étangs. L'objectif de ce stage était d'informer, de sensibiliser et de former des employés communaux des communes riveraines des plans d'eau et diverses 
personnes concernées, à un titre ou à un autre, par ces proliférations de plantes. Un des buts pratiques de cette formation de terrain était d'apprendre à reconnaître les espèces exotiques, de suivre leur extension et de les combattre avec des moyens limités, tels que l'arrachage manuel dans le cas de débuts d'invasions. Ces interventions ponctuelles mais régulières auraient pour but d'empêcher l'envahissement de nouveaux plans d'eau par les espèces proliférantes.

Une cinquantaine de personnes ont assisté à la journée en salle, plus particulièrement consacrée à la diffusion d'informations sur les plans d'eau et les difficultés particulières liées aux plantes aquatiques, et aux deux journées de terrain où des plantes ont été observées in situ, prélevées et déterminées. Des fiches photographiques présentant les principales espèces et surtout les plantes introduites ont été réalisées et distribuées à cette occasion.

Des visites spécialisées, consacrées aux problèmes spécifiques de divers plans d'eau, ont ensuite été faites durant l'été, avec les employés communaux et les représentants de la Pêche concernés.

\subsubsection{Suites de ce stage}

A l'heure actuelle, seules deux opérations peuvent être considérées comme des conséquences de ce stage. II s'agit de l'entretien régulier des plantes dans l'Etang de Moïsan et d'un arrachage de la jussie sur les rives de l'Etang Noir, classé en réserve naturelle.
Le dragage de l'Etang de Moïsan a fait disparaître momentanément les macrophytes présents, mais la recoIonisation rapide a conduit la municipalité à entretenir le plan d'eau: l'achat d'un bateau a permis à un employé municipal d'intervenir régulièrement pour arracher manuellement les herbiers de $M$. brasiliense en cours de reconstitution et contenir ainsi cette colonisation. Ces travaux d'entretien sont réalisés par une personne seule, pendant 2 à 3 demi-journées par trimestre.

L'arrachage de Ludwigia dans l'Etang Noir a été fait à l'occasion des Journées de l'Environnement de juin 1992 (Anonyme, 1992). II a consisté en l'enlèvement de deux herbiers denses d'une cinquantaine de mètres carrés. Le suivi que nous avons réalisé sur ce plan d'eau en 83,88 et 91 (Tableau $n^{\circ} 6$ ) nous avait montré la relative rapidité d'extension de cette espèce dans ce secteur (Dutartre, 1991), qui nous semblait devoir être nettoyé. Ces travaux d'arrachage manuel ont réunis une douzaine de bénévoles, membres d'associations de protection de la nature, de chasse et de pêche : en cela, leur valeur symbolique est beaucoup plus importante que leur efficacité.

\section{DISCUSSION}

Les opérations de gestion et d'entretien des plantes aquatiques réalisées dans le cadre du département des Landes rencontrent toujours de nom- 
Tableau 6. - Evolution de la colonisation de l'étang noir par Ludwigia entre 1983 et 1991

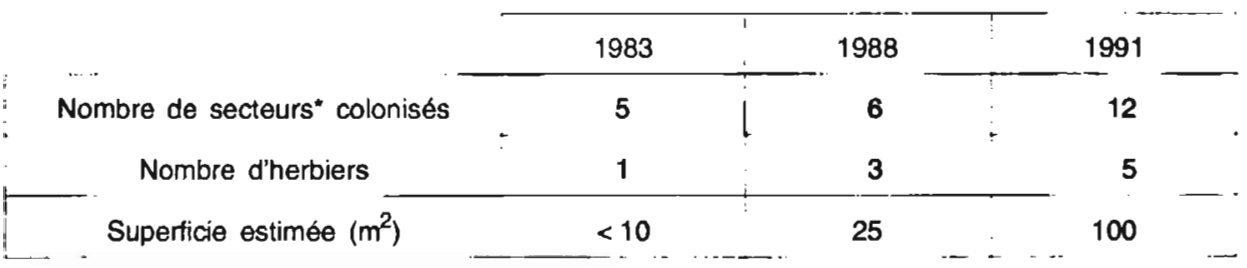

'(il s'agit de secteurs individualisés sur carte pour permettre une quantification de la répartition des plantes, 26 secteurs de référence couvrent l'ensemble du périmètre de l'étang)

breuses difficultés qui sont d'ordre conceptuels, écologique, social, économique et politique. Ces difficultés sont à la mesure de la complexité du problème posé que l'on pourrait décrire schématiquement de la manière suivante :

- les plans d'eau du littoral aquitain sont des écosystèmes fragiles, largement protégés par les dispositions réglementaires sur les sites, et classés en Zones Naturelles d'Intérêt Ecologique, Faunistique et Floristiques,

- ils constituent une ressource importante pour la région : ils en sont un des pôles d'attraction touristique et assurent indirectement d'importantes rentrées financières,

- les plantes aquatiques indigènes de ces lacs et étangs comportent des espèces rares ou endémiques, dont certaines sont protégées,

- certaines plantes aquatiques exotiques, apportées sciemment ou accidentellement par les hommes $y$ causent des désordres écologiques et fonctionnels vis à vis des activités humaines,

- des interventions sont nécessaires pour contrôler les nuisances ainsi créées, qui ne doivent pas altérer de façon irréversible les capacités de ces écosystèmes....

- ces interventions doivent être d'un coût supportable par les collectivités gestionnaires et donner des résultats visibles pour des non-initiés,

- pour les gestionnaires, il serait préférable qu'elles soient de l'investissement et non du fonctionnement.....

Dans ce contexte, une partie de l'argumentation développée par les scientifiques est une boucle logique: protéger les écosystèmes revient à leur conserver leurs potentialités à satisfaire les usages (le maximum d'usages), et a donc pour conséquence de protéger les activités touristiques.... Cet argument très approximatif est suivi de propositions de zonages ou d'organisation des activités... Mais la dimension écologique n'est pas encore vraiment prise en 
compte dans cette problématique: c'est un des seuils que les limnologues désireux d'intervenir dans la gestion des milieux doivent encore faire franchir. C'est ainsi, par exemple, que certaines plantes protégées, croissant dans des zones soumises à des projets d'aménagement touristiques, disparaissent sans qu'il leur soit prêté attention ou que les impacts des aménagements des rives ne peuvent pas toujours être prévu dans leurs détails.....

La gestion des plantes aquatiques, comme toute intervention de gestion sur des écosystèmes naturels, ne peut être autre chose qu'un entretien régulier, opération à répéter tant que les objectifs subsistent : par exemple, la moisson de L. major dans l'Etang Blanc est directement (et presque exclusivement) liée à la satisfaction des usages touristiques estivaux: chasseurs et pêcheurs locaux ne semblent pas trop se plaindre de cette prolifération.....

Cette dimension de "fonctionnement " est une contrainte financière très importante pour les gestionnaires qui attendent très souvent une solution définitive à leurs difficultés.... La demande en est évidemment faite aux techniciens et aux scientifiques qui doivent alors expliquer la complexité des interactions, la prudence à conserver dans les actions et les nécessités de suivre les évolutions des milieux traités.

Les techniciens sont également largement confrontés à des propositions techniques de sociétés, des articles de publications "grand public" ou des interventions médiatiques n'analysant pas complètement les problèmes rencontrés et proposant donc des solutions a priori "définitives" à des partenaires soucieux des dépenses publiques. Un exemple récent de cet aspect des choses est la demande de traitement des proliférations de $L$. major par la carpe chinoise. Dans un premier temps, nous avions écarté cette solution, mais, à la suite de questions provenant du Conseil Général, des essais avec ce poisson herbivore ont été envisagés. Ils devaient porter tout d'abord sur les choix alimentaires de l'espèce (c'est à dire "ce poisson consommerait-il seulement les plantes qui sont jugées nuisibles ou s'attaquerait-il aux espèces indigènes indispensables à l'équilibre des peuplements fauniques?»). A l'heure actuelle, ce projet est abandonné pour diverses raisons dont l'introduction interdite de cette espèce dans les eaux libres en France.

Comme nous l'avons déjà signalé, dans le cas du département des Landes, la création du Syndicat Mixte Géolandes a permis de mieux organiser la réflexion, le choix et la programmation des interventions; de plus les enveloppes financières importantes mises à la disposition de ces activités ont facilité la réalisation de travaux à la "bonne dimension".

Mais il subsiste diverses difficultés provenant des divergences d'appréciation sur les problèmes rencontrés. Par exemple, le stage de 1991 n'a pas donné tous les résultats escomptés, dans la mesure où seule une mu- 
nicipalité a considéré que la gestion des plantes dans son plan d'eau devait être intégrée aux travaux d'entretien communaux.

Des efforts importants en matière de formation et d'information des élus, des employés des services techniques et des populations doivent encore être faits pour que la prise en compte de ces difficultés de gestion soit correcte.

II subsiste également des difficultés techniques importantes (matériel incomplètement adapté, en particulier); des modes d'organisation de cette gestion restent à inventer et des marchés à assurer aux entreprises peu nombreuses qui peuvent réaliser un entretien régulier dans des conditions économiquement supportables pour elles et les donneurs d'ordres.

Enfin, il nous semble que la gestion de notre environnement passe obligatoirement par une meilleure intégration des techniciens et des scientifiques dans les structures des gestion pour que les objectifs de cette gestion à long terme des écosystèmes supports des activités humaines soient moins négligés.

\section{REMERCIEMENTS}

Une part importante des études récentes de la Division Qualité des Eaux du CEMAGREF de Bordeaux dans ce domaine a été financée par le Syndicat Mixte Géolandes.

\section{BIBLIOGRAPHIE}

Anonyme, 1975. Contrôle chimique de la végétation aquatique: traitement par le réglone des herbiers de Jussiae du port de Parentis (Landes) - Mai-Juin 1975, CTGREF, Groupement de Bordeaux, Section Qualité des Eaux, Pêche et Pisciculture.Compte rendu $n^{\circ} 2,15$ pages.

Anonyme, 1992. Compte rendu de l'opération d'arrachage de la jussie. 6 juin 1992. Sepan-Landes, Réserve Naturelle de l'Etang Noir, Seignosse (40). 3 pages.

Collectif, 1985. Etude écologique des Etangs de Moliets, Laprade, Moisan. 1982-1983. Propositions d'aménagements de l'Etang de Moisan. CEMAGREF, Groupement de Bordeaux, Division Qualite des Eaux. SRAE Aquitaine, GEREA. Etude $\mathrm{N}^{\circ} 22$, 168 pages + annexes.

Dubernet J.F., et al., 1992. Influence de la nature du sédiment sur la maitrise d'une plante aquatique (Lagarosiphon major (Ridley) Moss.) par le fluridone. Suivi en laboratoire. ANPP, $15^{e}$ Conférence du COLUMA, Versailles, 2, 3, 4 décembre 1992, 1117-1125.

Dulong J., Dutartre A., 1989. Les ceintures de végétation aquatique des lacs et étangs landais: les contraintes de gestion et les solutions proposées. 223-241. Colloque AFIE : "L'écologie en France. Des recherches aux applications: vers de nouvelles perspectives". Lyon, 23-25 novembre 1989, 496 pages.

Dutartre A. et al, 1986. Qualité des plans d'eau du littoral landais. CEMAGREF Groupement de Bordeaux, Division Qualité des Eaux. Etude $n^{\circ} 28$. 72 pages.

Dutartre A., 1978. Végétation aquatique. Plantes exotiques du Sud Ouest. Répartition actuelle et risques d'extension. CTGREF, Groupement de 
Bordeaux, Division Qualité des Eaux. Compte Rendu $n^{\circ} 45.24$ pages.

Dutartre A., 1979. Recherches préliminaires sur Lagarosiphon major (RIDLEY) MOSS. (Hydrocharidacée) dans le lac de Cazaux-Sanguinet-Biscarrosse. D.E.A. Université de Bordeaux III. Laboratoire de Botanique. 73 pages.

Dutartre A., 1989. Gestion des sédiments et des plantes aquatiques dans les lacs et étangs landais. in "La gestion environnementale des plans d'eau", IIGGE, Aix les Bains, 7 et 8 juin 1989. 13 pages.

Dutartre A, 1991. Suivi limnologique de l'Etang Noir. CEMAGREF Groupement de Bordeaux, Division Qualité des Eaux. Compte Rendu $n^{\circ} 81,10$ pages.
Dutartre A., sous presse. Gestion de la végétation aquatique. Prolifération de certaines espèces, nuisances induites et modes de contrôle. Actes des "Journées techniques sur les lacs et étangs aquitains ", 14-15 mai 1992.

Dutartre A., Delarche A., Dulong J., 1989. Gestion des plantes aquatiques dans les plans d'eau. Exemple des lacs et étangs landais. Info. Tech. CEMAGREF, 1989, No 76 , note 3, 8 pages.

Dutartre A., Delarche A., Dulong J., 1989. Végétation aquatique des lacs et étangs landais. Proposition d'un plan de gestion. CEMAGREF, Groupement de Bordeaux, Division QEPP, GEREA. Etude $n^{\circ} 38,121$ pages.

Sculthorpe C.D., 1967. The biology of aquatic vascular plants. ARNOLD Publ., London, 610 p. 